\title{
An algorithm and a method to search bifurcation points in non-linear problems
}

\author{
R. Chambon ${ }^{1, *, \dagger}$, S. Crochepeyre ${ }^{1}$ and R. Charlier ${ }^{2}$ \\ ${ }^{1}$ Laboratoire $3 S$ Grenoble Université Joseph Fourier, Institut National Polytechnique, C.N.R.S. U.M.R. 5521, \\ B.P. $53 X, 38041$ Grenoble Cedex, France \\ ${ }^{2}$ Département d'Infrastructure et de Géomécanique - DIG, Institut de Mécanique et Génie Civil-Bat. \\ B52/3 Université de Liége Chemin des Cheureucls, 1. 4000 Liége Sart Tilman, Belgique
}

\begin{abstract}
SUMMARY
This paper is devoted to a numerical method able to help the determination of the bifurcation threshold in non-linear time-independent continuum mechanic problems. First, some theoretical results about uniqueness are recalled. In the framework of the large-strain assumption, the differences between the classical finite-step problem and the rate problem are presented. An iterative algorithm able to solve the rate problem is given. Using different initializations, it is seen in some numerical experiments that it is possible with this algorithm to get different solutions when the underlying mathematical problem solved does not enjoy a uniqueness property. The constitutive equations used have been chosen to be simple enough to deduce some theoretical knowledge about the corresponding uniqueness problems. Finally, a method is given which is able in some case to give an upper bound of the bifurcation threshold. Copyright (C) 2001 John Wiley \& Sons, Ltd.
\end{abstract}

KEY WORDS: uniqueness; bifurcation; strain localization; buckling; large strain formulation; rate equilibrium problem; non-linearity

\section{INTRODUCTION}

A step-by-step procedure, and for every step, iterative algorithms are usually needed to solve time-independent non-linear problems. However, convergence of these algorithms is not a proof of the uniqueness of the problem and consequently, in some cases it is difficult to evaluate the validity of the found solutions. As we have to solve more and more non-linear problems which well posedness and consequently uniqueness are not proved and are then questionable, it can be interesting to know the bifurcation point, i.e. the threshold of the loading parameter which corresponds to the first loss of uniqueness. In fact, the main results about bifurcation have been proved for the rate problem only (recall that for the rate problem the unknown field is the velocity field). A spectral analysis has to be performed which turns out

\footnotetext{
${ }^{*}$ Correspondence to: R. Chambon, Laboratoire $3 \mathrm{~S}$ Grenoble Université Joseph Fourier, Institut National Polytechnique,C.N.R.S. U.M.R. 5521, B.P. 53X, 38041 Grenoble Cedex, France

†E-mail: rene.chambon@hmg.impg.fn
} 
into an eigenvalue problem for the corresponding finite element problem. If the rate problem is non-linear, the difficulties are larger. In the framework of elasto-plasticity, theoretical results gives bound criterion by the use of the so-called comparison solid theory given first by Hill for elasto-plastic materials obeying normality rule [1,2]. Extensions by Raniecki [3, 4] for one mechanism elasto-plastic materials which do not meet the normality condition provide a lower bound of the first critical bifurcation point. Numerical applications of the method finally yields to solve linear problems for the suitable linear comparison solid. In addition, it is possible to give for specific models an upper bound by the use of an other linearization of the model along the current loading direction given by the basic solution [5,6]. This also yields too to a linear analysis. Linearizing problems is also a current way to deal with more general bifurcation (see, for instance, the work of Loret and coworkers about flutter instability [7]).

Herein our purpose is to go further, in that we intend to deal with the complete nonlinear rate problem without any linearization. In fact, linearizing the problem has theoretical justification only in the few situations recalled above. In this paper, a method and an algorithm are presented which are able to give not only an upper bound of the bifurcation threshold but also different (if any) solutions of time-independent boundary value rate problems. The method is completely different from the ones based on the Hill work and finally does not use any comparison solid and any linear method. This method is model independent provided the models used are rate independent ones. In the case of elasto-plastic models, the upper bound found can be a best approximation since the internal boundary between the loading area and the unloading one can be completely independent for the different solutions found and different with the one of the basic fundamental solution usually used to get the classical upper bound [6]. It is worth noticing here that contrary to many studies, the problems solved are thoroughly non-linear problems.

It is our choice to develop this method in the framework of the so-called large-strain assumption. Otherwise, some important bifurcation phenomena such as buckling would not be exhibited such as the ones seen in Section 7.3. Moreover, once the equations are properly written it is not more difficult to deal with the large-strain problem than with the corresponding small strain one.

Finally, we present a way to follow different solutions for the same boundary value problem, after the bifurcation point in a step-by-step computation.

Many works have been done about post-localization problems which is a particular case of post-bifurcation. In this case, it is well known that it is necessary to enhance the constitutive model by introducing an internal length. Two main ways have been followed to solve this post localization problem. The first one is to put an embedded discontinuity inside the mesh of the numerical computation. Many work has been done in this spirit. Ortiz et al. [8], Belytschko et al. [9], Simo et al. [10], Armero et al. [11] and Larsson et al. [12] amongst others have worked about such improvement of finite element method. The reader is requested to see the review of Jirasek [13], for a more complete reference list. The other way is the use of enhanced models (non-local [14], Cosserat [15], or different kinds of second gradient models [16-22]). As for simplicity, in the applications given here, we do not want to use an enhanced model, we restrict our attention about post bifurcation to buckling problems. However, it is likely that the proposed method can be extend to enhanced models for post-localization computations.

Following this introduction, the second part of this paper is devoted to some basic statements about the classical finite step problem. The principle of a usual algorithm of a fully implicit method used to get the solution of one step is recalled. This allows to clarify the 
difference between this problem and to the rate problem which is the problem considered in uniqueness studies. The latter is presented in the third part of this paper. The fourth part is a description of the so-called directional searching algorithm, the central point of the presented method. A model used for the following numerical experiments is detailed in the fifth part. Some numerical experiments are described and discussed in the sixth part. The seventh part is a description of the proposed method. Examples and a discussions about post-localization modeling are given. Finally, some concluding remarks about the limitations and the possibilities of the proposed method are discussed.

Let us finally give the principles of our notations. A component is denoted by the name of the tensor (or vector) accompanied with tensorial indices. All tensorial indices are in lower position as there is no need in the following of a distinction between covariant and contravariant components. Upper indices have other meanings. Second-order tensors are denoted by the name of the tensor underlined, whereas the fourth-order tensors are denoted with a double underline. The summation convention with respect to repeated tensorial indices has been adopted. Dots as usual denote time derivatives. As far as the matrical notation are concerned, square matrix are denoted with square brackets and one-dimensional matrix with braces.

\section{THE FINITE STEP PROBLEM}

Let us consider the motion of a general body submitted to some boundary conditions during a loading process. This body can undergo large displacements and large strains, this means that its position and its shape evolve during the loading process. In the following, a stationary Cartesian co-ordinates system is considered to define the components of the mathematical quantities. The co-ordinates of a material point of the structure are expressed by $x_{i}^{t}$ in the configuration at time $t$. It should be noted that $t$ has to be regarded only as a convenient variable which refers to the magnitude of the loading, as for simplicity, time-dependent effects are not considered in the study to follow.

The equilibrium equations have to be satisfied throughout the complete loading history. Many formulations are available to write the equilibrium of the structure. The balance equations can be written for instance, in the current configuration. So, using the principle of virtual work, this expression of the equilibrium in the current configuration is: for all kinematically admissible field $\dot{u}^{*}$ :

$$
\int_{\Omega^{t}} \sigma_{i j}^{t} \frac{\partial \dot{u}_{i}^{*}}{\partial x_{j}^{t}} \mathrm{~d} \Omega^{t}-\int_{\Gamma_{\sigma}^{t}} T_{i}^{t} \dot{u}_{i}^{*} \mathrm{~d} \Gamma^{t}-\int_{\Omega^{t}} \rho^{t} f_{i}^{t} \dot{u}_{i}^{*} \mathrm{~d} \Omega^{t}=0
$$

where $\sigma_{i j}^{t}$ are the components of the Cauchy stress tensor at time $t, \dot{u}_{i}^{*}$ are the components of the field of virtual velocities kinematically admissible at any time $t, \Omega^{t}$ is the configuration at time $t, \Gamma_{\sigma}^{t}$ is part of the boundaries $\Gamma^{t}$ where the stress vector is known at time $t$. The other part of $\Gamma^{t}$ is called $\Gamma_{u}^{t}$. The virtual field kinematically admissible previously defined is such that $\dot{u}^{*}=0$ on $\Gamma_{u}^{t}$. $\Gamma^{t}$ is then defined such as $\Gamma_{u}^{t} \cup \Gamma_{\sigma}^{t}=\Gamma^{t}$ and $\Gamma_{u}^{t} \cap \Gamma_{\sigma}^{t}=\emptyset, T_{i}^{t}$ are the components of the forces per unit area of deformed body applied on the boundaries $\Gamma_{\sigma}^{t}$. They are assumed to be known at time $t, \rho^{t}$ is the mass density at time $t, f_{i}^{t}$ are the body forces per unit mass at time $t$.

Obviously, it is impossible to check Equation (1) numerically at every time of the loading process. So what people generally do is discretizing the loading process in finite time steps called $\Delta t$, and determining equilibrium positions of the volume for each time step. 
Let us consider a body which boundary conditions are applied on, at a given time $t$ of the loading process. This body is supposed to be well balanced. The corresponding configuration is called $\Omega^{t}$. The time-step problem consists in determining a position of the body that satisfies the equilibrium equation (1) at time $\tau=t+\Delta t$. Let us now define an approximation of the configuration at time $\tau=t+\Delta t$, called $\Omega^{\tau 1}$. Equilibrium equations are supposed not to be met in such a configuration. Thus, out of balance forces $F_{i}^{\text {obf }}$ can be defined such as, for all kinematically admissible field $\dot{u}^{*}$ :

$$
\int_{\Omega^{\tau 1}} \sigma_{i j}^{\tau 1} \frac{\partial \dot{u}_{i}^{*}}{\partial x_{j}^{\tau 1}} \mathrm{~d} \Omega^{\tau 1}-\int_{\Gamma_{\sigma}^{\tau 1}} T_{i}^{\tau 1} \dot{u}_{i}^{*} \mathrm{~d} \Gamma^{\tau 1}-\int_{\Omega^{\tau 1}} \rho^{\tau 1} f_{i}^{\tau 1} \dot{u}_{i}^{*} \mathrm{~d} \Omega^{\tau 1}=\int_{\Omega^{\tau 1}} F_{i}^{\mathrm{obf}} \dot{u}_{i}^{*} \mathrm{~d} \Omega^{\tau 1}
$$

The aim is now to determine another configuration called $\Omega^{\tau 2}$, assumed to be close to $\Omega^{\tau 1}$, such as, for the latter, the out of balance forces vanish. This leads then to, for all kinematically admissible field $\dot{u}^{*}$ :

$$
\int_{\Omega^{\tau 2}} \sigma_{i j}^{\tau 2} \frac{\partial \dot{u}_{i}^{*}}{\partial x_{j}^{\tau 2}} \mathrm{~d} \Omega^{\tau 2}-\int_{\Gamma_{\sigma}^{\tau 2}} T_{i}^{\tau 2} \dot{u}_{i}^{*} \mathrm{~d} \Gamma^{\tau 2}-\int_{\Omega^{\tau 2}} \rho^{\tau 2} f_{i}^{\tau 2} \dot{u}_{i}^{*} \mathrm{~d} \Omega^{\tau 2}=0
$$

Let us remark that, in the change of configuration, $\Gamma_{u}^{\tau 1}$ and $\Gamma_{\sigma}^{\tau 2}$ become, respectively, $\Gamma_{u}^{\tau 2}$ and $\Gamma_{\sigma}^{\tau 2}$. The set of virtual field kinematically admissible at time $\tau 1$ evolves to the set of virtual field kinematically admissible at time $\tau 2$. In order to get a proper linearization of the problem, we write Equation (3) in configuration $\Omega^{\tau 1}$ and subtract each term of the equation obtained from the corresponding term of Equation (2) and develop the three terms on the left-hand side in a Taylor expansion discarding terms of degree greater than 1. After some mathematics this yields to the following linear auxiliary problem, the unknown of which is the displacement field $\mathrm{d} u$ to be added to the current configuration $\Omega^{t}$. For all kinematically admissible field $\dot{u}^{*}$ :

$$
\int_{\Omega^{\tau 1}}\left[C_{i k j l}^{\mathrm{fs}} \frac{\partial \mathrm{d} u_{k}}{\partial x_{l}^{t}}+\sigma_{i k}^{\tau 1} \frac{\partial \mathrm{d} u_{l}}{\partial x_{l}^{\tau 1}}-\sigma_{i j}^{\tau 1} \frac{\partial \mathrm{d} u_{k}}{\partial x_{j}^{\tau 1}}\right] \frac{\partial \dot{u}_{i}^{*}}{\partial x_{k}^{\tau 1}} \mathrm{~d} \Omega^{\tau 1}-\int_{\Gamma_{\sigma}^{\tau 1}} \mathrm{~d} T_{i}(\mathrm{~d} u) \dot{u}_{i}^{*} \mathrm{~d} \Gamma^{\tau 1}=\int_{\Omega^{\tau 1}} F_{i}^{\mathrm{obf}} \dot{u}^{*} \mathrm{~d} \Omega^{\tau 1}
$$

where $\mathrm{d} T_{i}(\mathrm{~d} u)$ is the change of $T_{i}$ which can depend on the change of position of the boundary (if the boundary are prescribed following forces like pressure for instance), $C_{i k j l}^{\mathrm{fs}}$ is a tangent stiffness operator consistent with the algorithm used to compute the final stress of the current loading path. The practical importance of this consistent tangent stiffness has been illustrated by Simo et al. [23].

Finally, Equation (4) is the basis of a full Newton-Raphson method for a fully implicit solving of the time-step problem. Introducing the space discretization into finite elements, Equation (4) leads to a linear system to solve that can be written as follows:

$$
\left[K^{-\mathrm{fs}}\right]\{\mathrm{d} u\}=\left\{F^{\mathrm{obf}}\right\}
$$

where $\left[K^{\mathrm{fs}}\right]$ is the stiffness matrix including linear terms induced by large strains, $\{\mathrm{d} u\}$ are the nodal displacements correction, $\left\{F^{\text {obf }}\right\}$ are the out of balance forces at the nodes of the structure.

Let us notice that an assumption about the loading path between the beginning and the end of the time step is necessary for each Gauss point of the discretized body. Similarly, an integration method has to be chosen. The proper linearization of this integration method has to be performed. This gives the so-called consistent tangent stiffness matrix mentioned above. It is worth noticing too that the described method needs a first guess of the final (for the current 
time step) configuration of the body. Usually, this first guess is chosen as an extrapolation of the solution corresponding to the previous time step. But as we will see in Section 7, it can be interesting to use a completely different initial guess. A complete algorithm based on the previous principles can be seen for instance in the work of Charlier [24].

\section{THE RATE EQUILIBRIUM PROBLEM}

\subsection{The basic equations}

This section is devoted to the basic features of a different problem: the rate equilibrium problem. Theoretical studies about bifurcation are mainly loss of uniqueness for the rate problem. All the theoretical results quoted in the introduction are theorems about the rate problem. Let us define this boundary value problem. A well-balanced configuration $\Omega^{t}$ is assumed to be known. The rate of boundary conditions are assumed to be given and we are looking for the velocity field.

In order to get the corresponding balance equations in the virtual work form, let us consider the configuration $\Omega^{t}$ at time $t$ and another one $\Omega^{t+\delta t}$ at time $t+\delta t$. Equilibrium equations are supposed to be met in both cases. In order to get a proper linearization of the problem, balance equations corresponding to configuration $\Omega^{t+\delta t}$ are written in configuration $\Omega^{t}$ subtracted from balance equations corresponding to configuration $\Omega^{t}$. The result is developed in a Taylor expansion (where $\delta t$ is assumed to be small), terms of degree greater than one are discarded and velocities are introduced. After some mathematics detailed in the appendix, this yields to the following results. For any kinematically admissible field $\dot{u}^{*}$

$$
\begin{gathered}
\int_{\Omega^{t}}\left[\tilde{\sigma}_{i k}-\sigma_{m k}^{t} \omega_{m i}-\sigma_{i m}^{t} \omega_{m k}+\sigma_{i k}^{t} D_{l l}-\sigma_{i j}^{t}\left(D_{k j}+\omega_{k j}\right)\right] \frac{\partial \dot{u}_{i}^{*}}{\partial x_{k}^{t}} \mathrm{~d} \Omega^{t} \\
-\int_{\Gamma_{\sigma}^{t}} \dot{T}_{i}(\dot{u}) \dot{u}_{i}^{*} \mathrm{~d} \Gamma t-\int_{\Omega^{t}} \rho^{t} \dot{f}_{i}^{t} \dot{u}_{i}^{*} \mathrm{~d} \Omega^{t}=0
\end{gathered}
$$

where $D_{i k}$ and $\omega_{i k}$ are, respectively, the symmetric part and the skew-symmetric part of the velocity gradient. The boundary condition $\dot{T}_{i}(\dot{u})$ are assumed in the following to be linear with respect to the velocity field. An example of this situation can be encountered if these boundary conditions are given by a known pressure depending on the time (see the appendix for instance). Equation (5) can be rearranged as

$$
\int_{\Omega^{t}}\left[\tilde{\sigma}_{i k}-\sigma_{l k}^{t} \omega_{l i}+\sigma_{i k}^{t} D_{l l}-\sigma_{i j}^{t} D_{k j}\right] \frac{\partial \dot{u}_{i}^{*}}{\partial x_{k}^{t}} \mathrm{~d} \Omega^{t}-\int_{\Gamma_{\sigma}^{t}} \dot{T}_{i}(\dot{u}) \dot{u}_{i}^{*} \mathrm{~d} \Gamma t-\int_{\Omega^{t}} \rho^{t} \dot{f}_{i}^{t} \dot{u}_{i}^{*} \mathrm{~d} \Omega^{t}=0
$$

which is linear with respect to $\partial \dot{u}_{k} / \partial x_{j}^{t}$ except for the Jaummann stress rate $\underline{\tilde{\sigma}}$ which is generally non-linear if plastic effects have to be modelled. It is worth noticing however that this equation is positively homogeneous.

It is quite clear from the mathematical derivation of this section that the rate equilibrium problem can be seen as the limit of the problem corresponding to a step of vanishing magnitude in the framework of the finite step problem. 


\subsection{The iterative procedure}

Consider now a field $\dot{u}_{i}^{1}$ such as Equation (6) is not met. The rate of out of balance force $\dot{F}_{i}^{\text {obf }}$ is defined as follows. For any kinematically admissible field $\dot{u}^{*}$,

$$
\begin{aligned}
& \int_{\Omega^{t}}\left[\tilde{\sigma}_{i k}^{1}-\sigma_{l k}^{t} \omega_{l i}^{1}+\sigma_{i k}^{t} D_{l l}^{1}-\sigma_{i j}^{t} D_{k j}^{1}\right] \frac{\partial \dot{u}_{i}^{*}}{\partial x_{k}^{t}} \mathrm{~d} \Omega^{t} \\
& -\int_{\Gamma_{\sigma}^{t}} \dot{T}_{i}\left(\dot{u}^{1}\right) \dot{u}_{i}^{*} \mathrm{~d} \Gamma t-\int_{\Omega^{t}} \rho^{t} \dot{f}_{i}^{t} \dot{u}_{i}^{*} \mathrm{~d} \Omega^{t}=\int_{\Omega^{t}} \dot{F}_{i}^{\mathrm{obf}} \dot{u}_{i}^{*} \mathrm{~d} \Omega^{t}
\end{aligned}
$$

The aim is to find a field $\dot{u}_{i}^{2}$ assumed to be close (in a directional sense) to $\dot{u}_{i}^{1}$ such that the out of balance force rate vanishes. Then a proper linearization of Equation (7) allows us to get Equation (12). This needs a proper linearization of the rate constitutive equation. For time-independent constitutive equations, a directional linearization can be defined as follows: the relation between $\underline{\tilde{\sigma}}$ and $\underline{D}$ in the vicinity of a given strain direction $\operatorname{dir} \underline{D^{0}}=\underline{D}^{0} / \sqrt{D_{i j}^{0} D_{i j}^{0}}$ can be written as

$$
\tilde{\sigma}_{i j}=C_{i j k l}\left(\underline{\operatorname{dir} \underline{D}^{0}}\right) D_{k l}+t_{i j}
$$

where

$$
\lim _{\underline{\operatorname{dir} \underline{\underline{D}} \rightarrow \underline{\operatorname{dir}} \underline{\underline{D}}^{0}}} \frac{\underline{t}}{\left\|\underline{\operatorname{dir} \underline{D}}-\underline{\operatorname{dir} \underline{D}^{0}}\right\|}=0
$$

For instance, in the case of classical elasto-plastic models, we have

$$
C_{i j k l}\left(\underline{\operatorname{dir} \underline{D}^{0}}\right)=C_{i j k l}^{\mathrm{ep}}
$$

where $C_{i j k l}^{\mathrm{ep}}$ is the plastic tensor if $\underline{\operatorname{dir}} \underline{D}^{0}$ corresponds to a loading direction, and

$$
C_{i j k l}\left(\underline{\operatorname{dir} \underline{D}^{0}}\right)=C_{i j k l}^{\mathrm{e}}
$$

where $C_{i j k l}^{\mathrm{e}}$ is the elastic tensor if $\operatorname{dir} \underline{D}^{0}$ corresponds to an unloading direction. In Section 5, the directional linearization of the constitutive equation used in the presented computations is detailed. Finally, assuming that the body forces per unit mass are constant (i.e. independent of the position of the body), linearization of Equation (7) in the vicinity of a given rate field $\dot{u}_{i}^{1}$ such that $D_{i j}^{1}=\frac{1}{2}\left(\partial \dot{u}_{i}^{1} / \partial x_{j}^{t}+\partial \dot{u}_{j}^{1} / \partial x_{k}^{t}\right)$ yields Equation (12). For any kinematically admissible field $\dot{u}^{*}$ :

$$
\begin{gathered}
\int_{\Omega^{t}}\left[C_{i k j l}\left(\underline{\operatorname{dir} \underline{D}^{1}}\right) \mathrm{d} D_{j l}-\sigma_{l k}^{t} \mathrm{~d} \omega_{l i}+\sigma_{i k}^{t} \mathrm{~d} D_{l l}-\sigma_{i j}^{t} \mathrm{~d} D_{k j}\right] \frac{\partial \dot{u}_{i}^{*}}{\partial x_{k}^{t}} \mathrm{~d} \Omega^{t} \\
-\int_{\Gamma_{\sigma}^{t}} \mathrm{~d} \dot{T}_{i}(\mathrm{~d} \dot{u}) \dot{u}_{i}^{*} \mathrm{~d} \Gamma_{\sigma}^{t}=\int_{\Omega^{t}} \dot{F}_{i}^{\text {obf }} \dot{u}_{i}^{*} \mathrm{~d} \Omega^{t}
\end{gathered}
$$

Introducing the finite element discretization, expression (12) leads to a linear system to solve that can be written as

$$
\left[K^{\mathrm{re}}\right]\{\mathrm{d} \dot{u}\}=\left\{\dot{F}^{\mathrm{obf}}\right\}
$$


defining $\left[K^{\text {re }}\right]$ the stiffness matrix resulting from Equation (12) through the space discretization into finite elements. Linear terms induced by large strains are included in this matrix. $\{\mathrm{d} \dot{u}\}$ are the nodal increment of velocities $\left\{\dot{F}^{\text {obf }}\right\}$ are the out of balance force rate at the nodes of the structure given by Equation (7) through the space discretization.

It should be noted that the compliance matrix $C_{i j k l}^{\mathrm{fs}}$ and $C_{i j k l}\left(\underline{\operatorname{dir}} \underline{D}^{1}\right)$ introduced, respectively, in Equations (4) and (13) are defined in two different ways.

On one hand, $C_{i j k l}^{\mathrm{fs}_{\mathrm{s}}}$ is used in order to linearize the equation used to solve the finite step problem with an iterative procedure. This linearization is performed with respect to the end of the increment. $C_{i j k l}^{\mathrm{fs}}$ can in all cases be defined by a perturbation method [24]. For some constitutive equations and for some stress point algorithm, this matrix can be computed analytically (see Reference [23] for instance).

On the other hand, $C_{i j k l}\left(\operatorname{dir} \underline{D}^{1}\right)$ is used in order to linearize the equation used to solve the rate equilibrium problem with an iterative procedure. This linearization is performed with respect to $\partial \mathrm{d} \dot{u}_{k} / \partial x_{e}^{t}$. Thus, for elasto-plastic models as well as for incrementally non-linear models (under some conditions), $C_{i j k l}\left(\operatorname{dir} \underline{D}^{1}\right)$ can be defined in an analytical way by a directional linearization of the constitutive equation in the vicinity of a given strain rate. The resulting stiffness matrixes $\left[K^{\mathrm{fs}}\right]$ and $\left[K^{\mathrm{re}}\right]$ do obviously not coincide.

It is worth noticing in Equation (12), that besides terms involving the compliance matrix, the other terms are due to the large-strain assumption. It is consequently clear that a small-strain assumption is available provided the stress level is negligible with respect to the components of the compliance matrix.

\section{THE DIRECTIONAL SEARCHING ALGORITHM}

Similarly, to the case of the finite step problem, an iterative procedure is deduced from Equation (12) to solve the rate equilibrium problem which can be seen as a full NewtonRaphson method corresponding to the rate problem. In this case, the unknown of the problem are the nodal velocities whereas the configuration and the state of the structure remain constant. At the beginning of the algorithm, an assumption is performed on these nodal velocities which, as shown further, has a crucial influence on the results when uniqueness of the solution is lost. The different stages of the directional searching algorithm are presented in Table I. In order to check the accuracy of the solution at the end of each iteration, the angle between the assumed and the resulting nodal velocities directions (this means that nodal velocities of iteration $n-1$ and $n$ are compared) is computed for each node and the maximum value of this angle for all the nodes of the discretized problem is taken as convergence criterion. In the following, the algorithm has been used (as seen in Figure 3) up to the numerical noise which means with the computer used an angle between the two directions of about $10^{-6} \mathrm{deg}$.

\section{THE CONSTITUTIVE EQUATION USED}

All the following results are get with the same constitutive equation which has the advantages to be both simple enough to have a few number of parameters and versatile enough to exhibit or not localized solutions, according to the change of one parameter. Although this model is incrementally non-linear, complete theoretical shear band analysis can be performed. A study 
Table I. The directional searching algorithm.

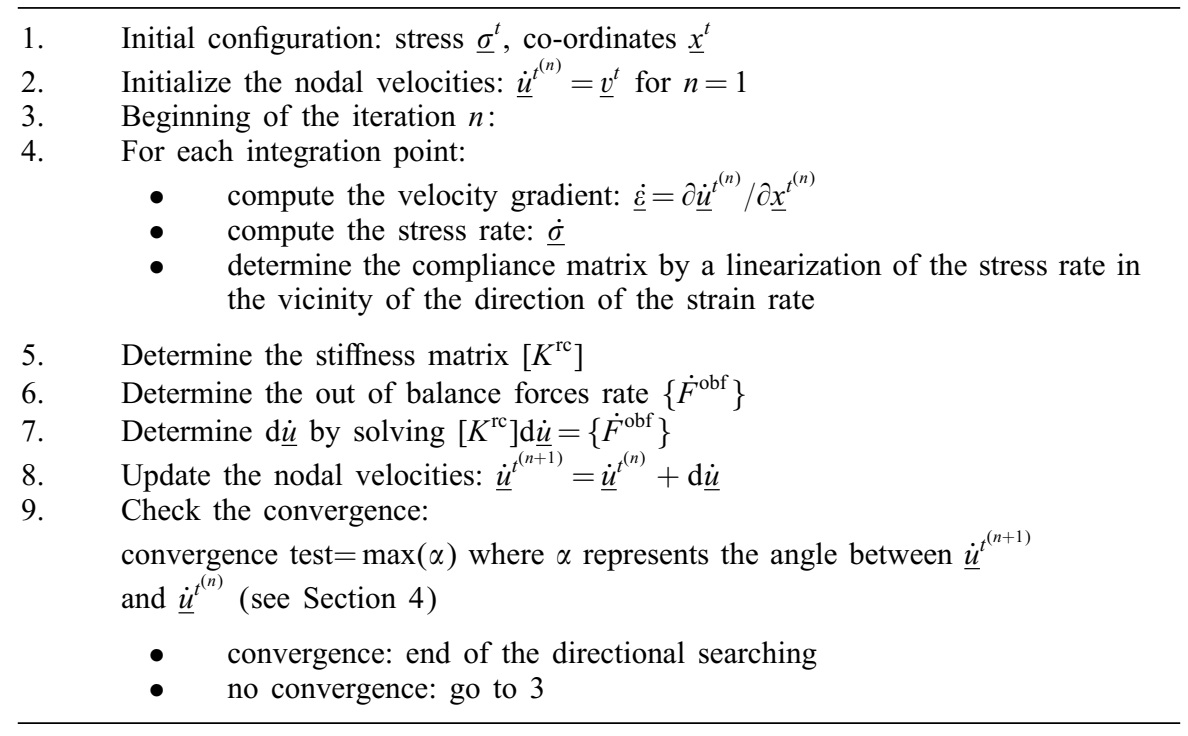

of the generic form of the model used here has already been done [25] and we used these results without proof. This class of constitutive equations named CLoE model is defined by the non-linear generic form

$$
\tilde{\sigma}_{i j}=A_{i j k l}\left(D_{k l}+b_{k l}^{\prime}\|\underline{D}\|\right)
$$

where $\tilde{\sigma}_{i j}$ is the Jaummann derivative of the Cauchy stress, $D_{k l}$ is the strain rate, $A_{i j k l}$ is a fourth-order tensor, and $b_{k l}^{\prime}$ is a second-order tensor. The behaviour of the material depends only on the second stress invariant. The limit surface is in that case represented in the principal stress space by a cylinder of radius centered on the mean pressure axis (von Mises-like model). $\underline{b^{\prime}}$ is chosen as a purely deviatoric tensor such that

$$
b_{k l}^{\prime}=\frac{2 G}{2 G+H} \bar{s}_{k l}
$$

where $G$ is analogue to an elastic shear modulus, $\bar{s}_{k l}$ is the normalized deviatoric stress defined with respect to the deviatoric stress $s_{k l}$ such as $\bar{s}_{k l}=s_{k l} /\|s\|$ and $H$ is a function of the state such that

$$
H=2 G \frac{\left(q-q^{\lim }\right)^{2}}{q\left(2 q^{\lim }-q\right)}
$$

where $q=\|s\|=\sqrt{s_{i j} s_{i j}}$ represents the second strain invariant. $q^{\text {lim }}$ represents the maximum value of $q$. Combining, Equations (16) and (15) yields

$$
b_{k l}^{\prime}=\hat{s}_{k l}\left(2-\frac{q}{q^{\lim }}\right)
$$

where $\hat{s}_{k l}$ is defined by $\hat{s}_{k l}=s_{k l} / q^{\lim }$.

The tensor $\underline{\underline{A}}$ is chosen as analogue as the fourth-order isotropic elastic tensor except for the out of axis (the axis are the principal axis of the stress) shear modulus. For any stress 


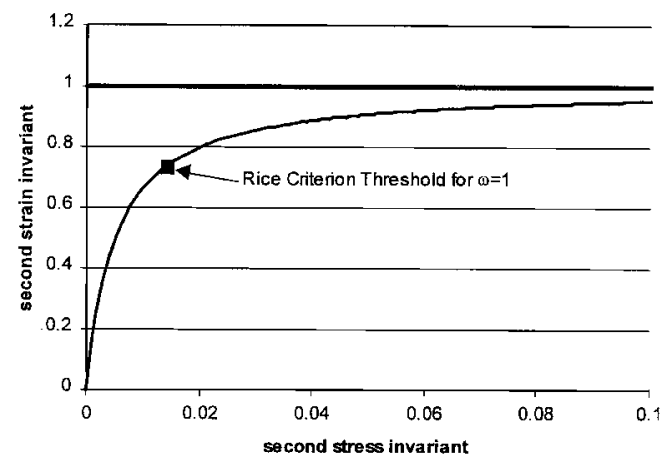

Figure 1. Stress-strain curve.

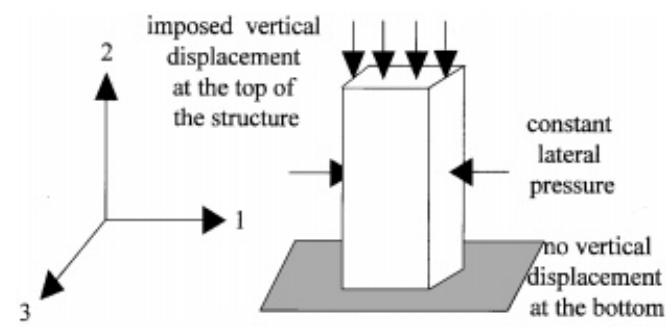

Figure 2. The biaxial test.

state, except the isotropic ones, a kind of anisotropy governed by the $\omega$ parameter has been introduced, in a similar way as we did [25], on the out of axis shear modulus $g$ in order to control the onset of localization

$$
g=G\left[1-\omega \frac{q}{q^{\lim }}\right]
$$

Under these assumptions, the model depends only on four parameters:

- $q^{\lim }$ chosen equal to 1 for simplicity.

- $K$ analogue to a bulk modulus.

- $G$ analogue to a shear modulus.

- $\omega$ in order to fit the onset of localization.

Thus, the tensor $\underline{\underline{A}}$ may be written in the case of plane strain in a matrix form as

$$
\underline{\underline{A}}=\left[\begin{array}{cccc}
K+\frac{4}{3} G & K-\frac{2}{3} G & K-\frac{2}{3} G & 0 \\
K-\frac{2}{3} G & K+\frac{4}{3} G & K-\frac{2}{3} G & 0 \\
K-\frac{2}{3} G & K-\frac{2}{3} G & K+\frac{4}{3} G & 0 \\
0 & 0 & 0 & 2 g
\end{array}\right]
$$

In a one-dimensional case, the evolution of the second stress invariant versus the second strain invariant is presented in Figure 1. We [26] have shown that the CLoE models can be directionally linearized in the vicinity of a given direction $\operatorname{dir} \underline{D^{1}}$, and we have

$$
C_{i j k l}\left(\underline{\operatorname{dir} \underline{D}^{1}}\right)=A_{i j k l}+A_{i j m n} b_{m n}^{\prime} \frac{D_{k l}^{1}}{\left\|\underline{D}^{1}\right\|}
$$

Finally, an analytical shear band analysis in the sense of Rice [27] can be performed with the CloE model, yielding to a non-linear criterion [28]. It is worth noticing that if $\omega=0$ then localization is only possible for stress states belonging to the limit surface (surface splitting the stress space between admissible states and impossible ones) which is here obviously a von Mises surface defined by $q^{\lim }=1$. On the contrary, when $\omega=1$ shear banding is possible for a value of the second invariant $q$ less than 1 as it can be seen in Figure 1. 
Table II. Results of the directional searching algorithm.

\begin{tabular}{|c|c|c|c|c|}
\hline No. of DOF & $\begin{array}{c}\text { Material } \\
\text { parameters }\end{array}$ & $\begin{array}{l}\text { Initial } \\
\text { state }\end{array}$ & Initialization & $\begin{array}{c}\text { Resulting } \\
\text { bifurcation }\end{array}$ \\
\hline 383,1427 or 5495 & $\begin{array}{c}K=G=100 \\
\omega=0\end{array}$ & Homogeneous & $\begin{array}{l}\text { Homogeneous } \\
\text { or shear band }\end{array}$ & Homogeneous \\
\hline 383,1427 or 5495 & $\begin{array}{c}K=G=100 \\
\omega=1\end{array}$ & Homogeneous & Homogeneous & Homogeneous \\
\hline 383 & $\begin{array}{c}K=G=100 \\
\omega=1\end{array}$ & Homogeneous & Shear band & $\begin{array}{l}\text { Shear band from } \\
2.16 \text { per cent }\end{array}$ \\
\hline 1427 & $\begin{array}{c}K=G=100 \\
\omega=1\end{array}$ & Homogeneous & Shear band & $\begin{array}{c}\text { Shear band from } \\
1.79 \text { per cent }\end{array}$ \\
\hline 5495 & $\begin{array}{c}K=G=100 \\
\omega=1\end{array}$ & Homogeneous & Shear band & $\begin{array}{l}\text { Shear band from } \\
1.65 \text { per cent }\end{array}$ \\
\hline
\end{tabular}

\section{NUMERICAL EXPERIMENTS}

\subsection{The boundary value problems solved}

The following simulations deal with a plane strain biaxial compression test shown in Figure 2 . The sample is supposed to have the shape of a parallelepiped and is supposed to be initially isotropic. The given boundary conditions are

- a constant lateral pressure on the vertical boundaries,

- the vertical displacements of the top of the structure,

- no vertical displacement at the bottom of the structure,

- perfect sliding is assumed on the top and on the bottom,

- direction 3 is the plane strain direction.

In order to define the mesh, a preliminary bifurcation analysis is carried out to determine the direction of the first possible shear band. Hence, a biased line is introduced into the mesh in order to allow a discontinuity of the kinematics. In fact, in the case of initially homogeneous sample, the locus of the biased line determines the area where the shear band can take place. For the simulations presented in the following, several mesh refinements are used. The sample has a size of $170 \mathrm{~mm} \times 100 \mathrm{~mm}$. The aspect ratio of 1.7 is small enough to avoid buckling before localization (see Table II). Computations have been done for meshes with 383, 1427 and 5495 degrees of freedom (DOFs).

\subsection{Preliminary results}

Simulations presented in the present part are performed on an initially homogeneous sample. Thus, considering the assumptions about the biaxial test presented in Section 6.1, the homogeneous solution is always available.

Numerous numerical experiments using the directional searching algorithm are summarized in Table II. A step-by-step computation has been done and for every final state, the directional 


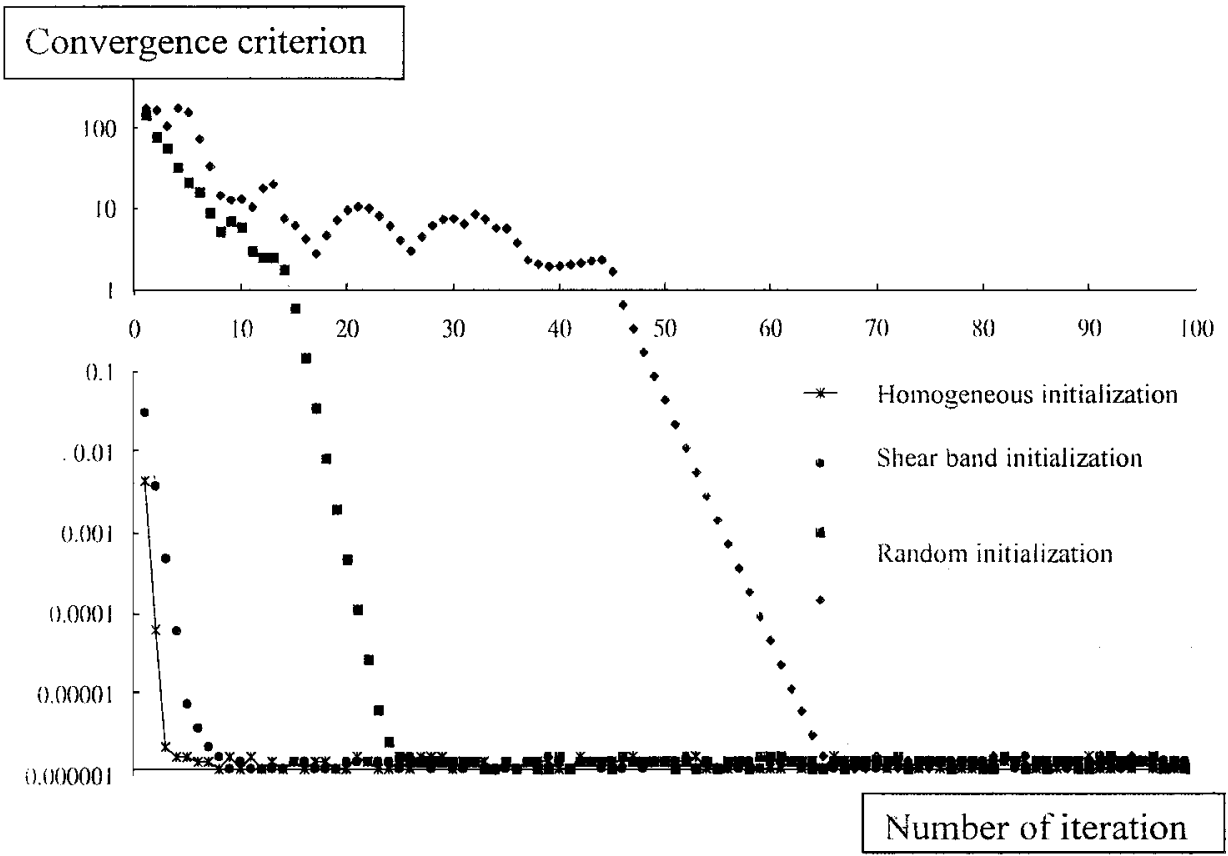

Figure 3. Convergence profile of the research directional algorithm.

searching algorithm is used to solve the rate problem. The step size is $1.25 \times 10^{-2} \mathrm{~mm}$ which means 136 time steps for an axial strain of 1 per cent. Every time step is converged up to the corresponding numerical noise. Two different initialization velocity fields are used. One of them correspond to an homogeneous strain field and another to a velocity field corresponding to a shear band, the position of which correspond to the biased line of the mesh.

The first firm result which have to be noticed is that the algorithm has always converged. Second, it is clear that as for classical problems there is a mesh dependency of the results. But the most important result is that in the case of theoretically non uniqueness, some different solutions can be reached by the way of different initial guess for the algorithm. Obviously, in the case of theoretical uniqueness, the (unique) solution is attained for any initialization (see the first row of Table II). Coming back to the convergence, it is interesting to have a look to Figure 3, where convergence profiles are plotted. The mesh is the 383 DOF one, the initial state correspond to 2.19 per cent of axial strain, and $\omega=1$. Four different initializations have been used, the two discussed above plus two random ones. For one of the random initialization, the converged solution is the homogeneous one and for the other initialization, the converged solution is the localized one.

Other computations, some with other constitutive equations like an elastoplastic softening one, have yet been published $[29,30]$. 
Table III. Results of the directional searching algorithm using a random initialization in the case of a $170 \mathrm{~mm} \times 100 \mathrm{~mm}$ sample discretized by means of 383 DOFs.

\begin{tabular}{|c|c|c|c|}
\hline \multirow[b]{2}{*}{$\begin{array}{l}\text { Initial state } \\
\text { (per cent) }\end{array}$} & \multicolumn{3}{|c|}{ Resulting mode } \\
\hline & $\begin{array}{l}\text { Homogeneous } \\
\text { (per cent) }\end{array}$ & $\begin{array}{l}\text { Shear band mode } 1^{*} \\
\text { (per cent) }\end{array}$ & $\begin{array}{c}\text { Shear band mode } 2^{\dagger} \\
\text { (per cent) }\end{array}$ \\
\hline 2.11 & 100 & - & - \\
\hline 2.19 & 38 & 52 & 10 \\
\hline 2.34 & 14 & 67 & 19 \\
\hline
\end{tabular}

* The shear band mode 1 is located above the biased line.

†The shear band mode 2 is located under the biased line.

\section{A NEW METHOD TO SEARCH THE NON-UNIQUENESS}

\subsection{The method: a random initialization of the directional searching algorithm}

Table III deals with the results of random initializations of the directional searching algorithm in the case of a sample discretized with 383 DOFs. The algorithm is initialized randomly for three different initial states. Twenty-one sequences of initial random velocity fields are tested. It is worth noticing that the velocity fields have to be consistent with the known velocities (the ones given by the boundary conditions). The first column of this table expresses the initial state in term of axial deformation. The second, third and fourth columns indicate the resulting modes obtained for each initial state and their corresponding ratio. For example, this means that for the initial state corresponding to a 2.19 for 38 out of 100 cases studied, whereas the first localized mode was obtained for 52 out of 100 cases. The second localized mode was obtained in the remaining 10 cases. So the proposed method to get (eventually) several solutions is to initialize the directional algorithm by random (with the above restrictions) velocity fields.

From the inspection of the result of Table III, it appears clearly that this method is able to detect the non-uniqueness of a problem. In this case, we know (using a complete Rice shear band analysis [28]) that the uniqueness of the mathematical underlying problem is lost for an axial strain equal to 1.39 per cent. It is obvious that the method does not give the true mathematical threshold, but an approximation of this value depending on the mesh. It is the same situation as for linear problems. In this latter case, it is well known that mesh influences the eigenvalues of a given problem. Other simple numerical experiences showing two differently oriented shear bands are reported elsewhere [28].

\subsection{Discussion}

As far as the directional searching algorithm is concerned, a great influence of the kinematic initialization was observed. In the presented example, three different types of solutions appeared: the homogeneous one and two localized ones. These three modes can then be regarded as fixed points of the algorithm. As these fixed points are reached for initializations different from the solutions, it can be said moreover that these fixed points are stable with respect to the used algorithm. A good way of understanding what happens it to compare the described numerical results to dynamical systems, and pertinent notions are then the ones of attractors 
and basins of attraction used for dynamical systems and finite-dimensional maps [31]. For small axial strains, the (here numerical) system has only one stable attractor: the velocity field corresponding to an homogeneous straining. Then after the threshold of bifurcation is encountered, other stable attractors appear which here correspond to velocity fields involving a shear band. The algorithm can give as output any of these stable attractors depending on the initialization. In the initialization space, we can define the basin of attraction of a solution as the locus of initializations leading to this solution. Table III shows that as the axial strain increases, the basins of attraction of the localized solutions likely grow.

Indeed, as already mentioned in Section 3, the directional searching algorithm can be considered as a limit of the finite step algorithm for a infinitesimal step. Consequently, it is possible to generalize the present idea and to used it in the finite step problem, for very small steps, a random initialization consistent with this loading step (which means for instance that the known values of the displacement on some boundary conditions have not to be randomized).

\subsection{The post bifurcation problem}

In this paper, for simplicity, we do not want to extend our work to cases for which we need a change of the model after bifurcation. So the presented application of post bifurcation problem is not about localized modes. We turn now our interest on buckling bifurcation which does not need any enhancement of the model, in the post bifurcation regime. The method described in Section 7.1 does not depend on the bifurcation mode and can then be used for buckling like modes.

In fact, the rate problem can be seen as the limit case of the finite step problem for a vanishing time-step duration. Thus there is a clear extension of the method described in Section 7.1 to the finite step algorithm. In the case of possible bifurcation, initializing with different initial guesses a finite step algorithm can yield different solutions.

Figure 4 presents result about computations of the biaxial test described in Section 6.1 using the models described in Section 5. The parameters are the same except that: $\omega=0$ which as seen in Section 5 prevents possible localizations in the model. However, the dimensions of the sample are now equal to $330 \mathrm{~mm} \times 100 \mathrm{~mm}$, which gives a slenderness of 3.3 .

The simulation presented here have been done for a mesh with 655 DOFs. The finite step algorithm has been used. The loading steps correspond to a vertical displacement of the top of the sample equal to $0.125 \mathrm{~mm}$. For some state (i.e. for some given global top displacement) the finite step algorithm is initialized with a buckling mode displacement field deduced from a solution found with the directional algorithm.

Up to a given point, the algorithm converges towards a homogeneous state, whatever the initialization. However, after some critical value, the converged solution of the finite step algorithm depends on the initialization. For a homogeneous initialization, the resulting solution is homogeneous. However, initializing with the buckling like field obtained by the method described in Section 7.1 yields a buckling (stable in the algorithm sense) solution. This means that a bifurcation mode detected by the present method can be followed by a finite step computation.

This way can be seen as an extension of the post bifurcation computations done by de Borst except that the injected mode was in the de Borst [32] study deduced from a linearized analysis. When the solution of a finite step has converged towards either the homogeneous 


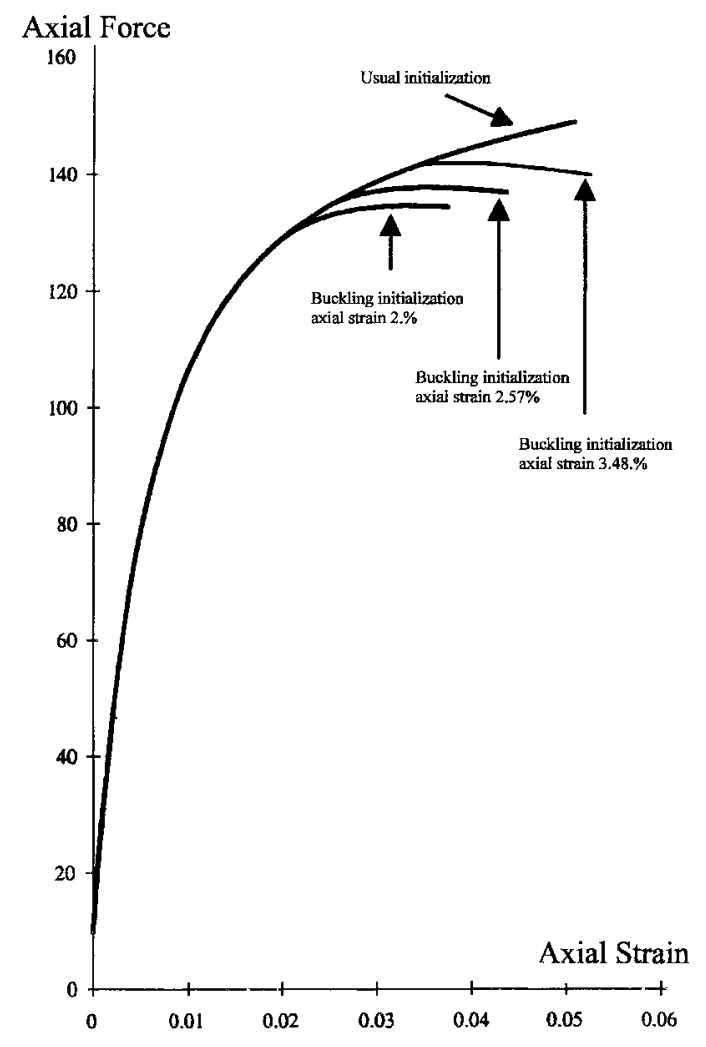

Figure 4. Different loading paths followed for the same problem.

solution or the buckling mode, the usual initialization (i.e. the continuation of the current displacement field) is used. In Figure 4, where the global force is plotted against the global displacement, it can be seen that it is possible to follow many different loading paths which are all different solutions of the same initial boundary value problem solved by a finite step method.

\section{CONCLUSION}

It has been demonstrated in this paper that it is possible from a numerical point of view to deal with the non-uniqueness of solutions of a boundary value problem without any linearization. Iterative resolutions of problems can be regarded in terms of dynamic systems behaviour. Concepts of attractors and basins of attraction seem to be pertinent for such studies. Basins of attraction (in the sense of the algorithm) evolve during the loading. If for a given state basin of attraction are great enough, then the proposed random initialization method allows us to discover an alternative solution for the rate problem (or for a given step of the finite step problem provided this step is small enough).

Moreover, this alternative solution given to the finite step algorithm as first guess can be used to follow different bifurcation branches. In the case of multiple solutions, the problem 
is then to choose one of them and then to find which one is the physical one. This study which in some case has been done for post-localization (see the references quoted in the introduction) is out of the scope of this paper and has obviously to be addressed in the future.

The method can be generalized to other non-linear field problems solved by the finite element method. This has been already done for second gradient models. It is likely that it can be generalized to other problems such as time-dependant ones.

\section{APPENDIX}

This appendix is devoted to the mathematical developments performed in order to obtain Equation (5). As mentioned in Section 3, Equation (5) is obtained by considering the equilibrium equations at time $t$ and $t+\delta t$. Thus, for any kinematically admissible field $\dot{u}_{i}^{*}$, we have at time $t$ :

$$
\int_{\Omega^{t}} \sigma_{i j}^{t} \frac{\partial \dot{u}_{i}^{*}}{\partial x_{j}^{t}} \mathrm{~d} \Omega^{t}-\int_{\Gamma_{\sigma}^{t}} T_{i}^{t} \dot{u}_{i}^{*} \mathrm{~d} \Gamma^{t}-\int_{\Omega^{t}} \rho^{t} f_{i}^{t} \dot{u}_{i}^{*} \mathrm{~d} \Omega^{t}=0
$$

and at time $t+\delta t$.

$$
\int_{\Omega^{t+\delta t}} \sigma_{i j}^{t+\delta t} \frac{\partial \dot{u}_{i}^{*}}{\partial x_{j}^{t+\delta t}} \mathrm{~d} \Omega^{t+\delta t}-\int_{\Gamma_{\sigma}^{t+\delta t}} T_{i}^{t+\delta t} \dot{u}_{i}^{*} \mathrm{~d} \Gamma^{t+\delta t}-\int_{\Omega^{t+\delta t}} \rho^{t+\delta t} f_{i}^{t+\delta t} \dot{u}_{i}^{*} \mathrm{~d} \Omega^{t+\delta t}=0
$$

The procedure consists in writing Equation (A2) in configuration $\Omega^{t}$ and subtracting each term of the resulting equation from the corresponding term of Equation (A1)

- For the first term:

$$
\int_{\Omega^{t+\delta t}} \sigma_{i j}^{t+\delta t} \frac{\partial \dot{u}_{i}^{*}}{\partial x_{j}^{t+\delta t}} \mathrm{~d} \Omega^{t+\delta t}-\int_{\Omega^{t}} \sigma_{i j}^{t} \frac{\partial \dot{u}_{i}^{*}}{\partial x_{j}^{t}} \mathrm{~d} \Omega^{t}=\int_{\Omega^{t}}\left[\sigma_{i j}^{t+\delta t} \frac{\partial x_{j}^{t}}{\partial x_{j}^{t+\delta t}} \operatorname{det}(J)-\sigma_{i k}^{t}\right] \frac{\partial \dot{u}_{i}^{*}}{\partial x_{k}^{t}} \mathrm{~d} \Omega^{t}
$$

where $\operatorname{det}(J)$ is the volumetric transformation between configurations $\Omega^{t}$ and $\Omega^{t+\delta t}$ defined as follows:

$$
\mathrm{d} \Omega^{t+\delta t}=\operatorname{det}(J) \mathrm{d} \Omega^{t}
$$

Let us define the displacement vector $\delta u$ between the initial and the final configuration such as

$$
x_{i}^{t+\delta t}=x_{i}^{t}+\delta u_{i}
$$

Combining Equations (A4) and (A5) leads to

$$
\int_{\Omega^{t}}\left[\sigma_{i j}^{t+\delta t}\left(\delta_{j k}-\frac{\partial \delta u_{k}^{t+\delta t}}{\partial x_{j}^{t+\delta t}}\right) \operatorname{det}(J)-\sigma_{i k}^{t}\right] \frac{\partial \dot{u}_{i}^{*}}{\partial x_{k}^{t}} \mathrm{~d} \Omega^{t}
$$

or, written in another way

$$
\int_{\Omega^{t}}\left[-\sigma_{i j}^{t+\delta t} \frac{\partial \delta u_{k}^{t+\delta t}}{\partial x_{j}^{t+\delta t}} \operatorname{det}(J)+\sigma_{i k}^{t+\delta t} \operatorname{det}(J)-\sigma_{i k}^{t}\right] \frac{\partial \dot{u}_{i}^{*}}{\partial x_{k}^{t}} \mathrm{~d} \Omega^{t}
$$


Defining by $\delta \sigma_{i k}$ and $\dot{\sigma}_{i k}$ the quantities such as

$$
\begin{aligned}
\sigma_{i k}^{t+\delta t} & =\sigma_{i k}^{t}+\delta \sigma_{i k} \\
\dot{\sigma}_{i k} & =\lim _{\delta t \rightarrow 0} \frac{\delta \sigma_{i k}}{\delta t}
\end{aligned}
$$

similarly

$$
\dot{u}_{i}=\lim _{\delta t \rightarrow 0} \frac{\delta u_{i}}{\delta t}
$$

Considering that $\delta t$ tends to zero and cancelling the terms of order higher than 1 , expression (A7) divided by $\delta t$, using Equations (A8)-(A10) yields

$$
\int_{\Omega^{t}}\left[\dot{\sigma}_{i k}+\sigma_{i k}^{t} \frac{\partial \dot{u}_{l}}{\partial x_{l}^{t}}-\sigma_{i j}^{t} \frac{\partial \dot{u}_{k}}{\partial x_{j}^{t}}\right] \frac{\partial \dot{u}_{i}^{*}}{\partial x_{k}^{t}} \mathrm{~d} \Omega^{t}
$$

Moreover, let us express $\partial \dot{u}_{k} / \partial x_{j}^{t}$ as the sum of its symmetric and skew-symmetric parts so that

$$
\frac{\partial \dot{u}_{k}}{\partial x_{j}^{t}}=D_{k j}+\omega_{k j}
$$

where $\omega_{i k}$ is the rotation rate and $D_{k j}$ is the so-called pure deformation rate.

The Jaummann stress rate is defined by

$$
\tilde{\sigma}_{i k}=\dot{\sigma}_{i k}+\sigma_{j k} \omega_{j i}+\sigma_{i j} \omega_{j k}
$$

Expression (A11) then becomes

$$
\int_{\Omega^{t}}\left[\tilde{\sigma}_{i k}-\sigma_{m k}^{t} \omega_{m i}-\sigma_{i m}^{t} \omega_{m k}+\sigma_{i k}^{t} D_{l l}-\sigma_{i j}^{t}\left(D_{k j}+\omega_{k j}\right)\right] \frac{\partial \dot{u}_{i}^{*}}{\partial x_{k}^{t}} \mathrm{~d} \Omega^{t}
$$

- For the second term:

This part is devoted to the computation of $\int_{\Gamma_{\sigma}^{t}} \dot{T}_{i}(\dot{u}) \dot{u}_{i}^{*} \mathrm{~d} \Gamma^{t}$ in a particular case. We assume that external tensions are only due to pressures. For every time $t$, we have

$$
T_{i}^{t}=p^{t} n_{i}^{t}
$$

This leads to

$$
\int_{\Gamma_{\sigma}^{t+\delta t}} p^{t+\delta t} n_{i}^{t+\delta t} \dot{u}_{i}^{*} \mathrm{~d} \Gamma^{t+\delta t}-\int_{\Gamma_{\sigma}^{t}} p^{t} n_{i}^{t} \dot{u}_{i}^{*} \mathrm{~d} \Gamma^{t}=\int_{\Gamma_{\sigma}^{t}}\left[p^{t+\delta t} n_{i}^{t+\delta t} \frac{\mathrm{d} A^{t+\delta t}}{\mathrm{~d} A^{t}}-p^{t} n_{i}^{t}\right] \dot{u}_{i}^{*} \mathrm{~d} \Gamma^{t}
$$

where $n_{i}^{t}$ represents the outward normal to $\Gamma_{\sigma}^{t}$ and $\mathrm{d} A^{t}$ the corresponding area. Expression (A16) can be rewritten as

$$
\int_{\Gamma_{\sigma}^{t}}\left[p^{t+\delta t} n_{i}^{t+\delta t} \mathrm{~d} A^{t+\delta t}-p^{t} n_{i}^{t} \mathrm{~d} A^{t}\right] \frac{\dot{u}_{i}^{*}}{\mathrm{~d} A^{t}} \mathrm{~d} \Gamma^{t}
$$

Using the Nanson formulae

$$
p^{t+\delta t} n_{i}^{t+\delta t} \frac{\mathrm{d} A^{t+\delta t}}{\mathrm{~d} A^{t}}=p^{t+\delta t} n_{j}^{t} \operatorname{det}(J) \frac{\mathrm{d} x_{j}^{t+\delta t}}{\mathrm{~d} x_{i}^{t}}
$$


Introducing the displacement vector between both configurations, dividing by $\delta t$ and linearizing like in the first part yields

$$
\int_{\Gamma_{\sigma}^{t}} \dot{T}_{i}(\dot{u}) \dot{u}_{i}^{*} \mathrm{~d} \Gamma^{t}=\int_{\Gamma_{\sigma}^{t}}\left[\dot{p} n_{i}^{t}+p^{t}\left[\frac{\partial \dot{u}_{j}}{\partial x_{i}^{t}} n_{j}^{t}+\frac{\partial \dot{u}_{l}}{\partial x_{l}^{t}} n_{i}^{t}\right]\right] \dot{u}_{i}^{*} \mathrm{~d} \Gamma^{t}
$$

- For the third term:

Using Equation (A4), the difference between the third term of the equilibrium equations written at time $t$ and $t+\delta t$ can be written in configuration $\Omega^{t}$ as

$$
\int_{\Omega^{t+\delta t}} \rho^{t+\delta t} f_{i}^{t+\delta t} \dot{u}_{i}^{*} \mathrm{~d} \Omega^{t+\delta t}-\int_{\Omega^{t}} \rho^{t} f_{i}^{t} \dot{u}_{i}^{*} \mathrm{~d} \Omega^{t}=\int_{\Omega^{t}} \rho^{t}\left[f_{i}^{t+\delta t}-f_{i}^{t}\right] \dot{u}_{i}^{*} \mathrm{~d} \Omega^{t}
$$

Dividing (A21) by $\delta t$ yields, as $\delta t$ tends to zero

$$
\int_{\Omega^{t}} \rho^{t} \dot{f}_{i}^{t} \dot{u}_{i}^{*} \mathrm{~d} \Omega^{t}
$$

Finally, provided expressions (A14), (A19) and (A21), the general equation of the problem can be written as

$$
\begin{array}{r}
\int_{\Omega^{t}}\left[\tilde{\sigma}_{i k}-\sigma_{m k}^{t} \omega_{m i}-\sigma_{i m}^{t} \omega_{m k}+\sigma_{i k}^{t} D_{l l}-\sigma_{i j}^{t}\left(D_{k j}+\omega_{k j}\right)\right] \frac{\partial \dot{u}_{i}^{*}}{\partial x_{k}^{t}} \mathrm{~d} \Omega^{t} \\
\int_{\Gamma_{\sigma}^{t}}\left[\dot{p} n_{i}^{t}+p^{t}\left[\frac{\partial \dot{u}_{j}}{\partial x_{i}^{t}} n_{j}^{t}+\frac{\partial \dot{u}_{j}}{\partial x_{i}^{t}} n_{i}^{t}\right]\right] \dot{u}_{i}^{*} \mathrm{~d} \Gamma^{t}-\int_{\Omega^{t}} \rho^{t} \dot{f}_{i}^{t} \dot{u}_{i}^{*} \mathrm{~d} \Omega^{t}=0
\end{array}
$$

\section{REFERENCES}

1. Hill R. A general theory of uniqueness and stability in elastic-plastic solids. Journal of the Mechanics and Physics of Solids 1958; 6:236-249.

2. Hill R. Aspect of invariance in solids. Advances in Applied Mechanics, Vol. 18. Academic Press: New York, $1978 ; 1-75$.

3. Raniecki B. Uniqueness criteria in solids with non-associated plastic flow laws at finite deformations. Bull. Acad. Pol. Sci. Tech. 1979; 27:391-399.

4. Raniecki B, Bruhns OT. Bounds to bifurcation stresses in solids with non associated plastic flow rule at finite strain. Journal of the Mechanics and Physics of Solids 1981; 29:153-172.

5. Kleiber M. On plastic localization and failure in plane strain and round void containing tensile bars. International Journal of Plasticity 1984; 2:205-221.

6. Tvergaard V. On bifurcation and stability under elastic-plastic deformation. In Plasticity Today Modeling, Methods and Applications, Sawscuck, Bianchi (eds). Elsevier: Amsterdam, 1985; 277-298.

7. Loret B, Martins J, Simoes F. Surface boundary conditions trigger flutter instability in non associative elastic plastic solids. International Journal of Solids and Structures 1995; 32:2155-2190.

8. Ortiz M, Leroy Y, Needleman A. A finite element method for localized failure analysis. Computer Methods in Applied Mechanics and Engineering 1987; 61:189-214.

9. Belytschko T, Fish J, Engelmann BE. A finite element with embedded localization zones. Computer Methods in Applied Mechanics and Engineering 1988; 70:59-89.

10. Simo J, Oliver J, Armero F. An analysis of strong discontinuities induced by strain softening in rate independant inelastic solids. Computational Mechanics 1993; 12:227-296.

11. Armero F, Garikipati K. Recent advances in the analysis and numerical simulation of strain localization in inelastic solids. Fifth International Conference on Computational Plasticity, Owen, Onate Hinton (eds). CIMNE, Barcelona 1996; 674-681.

12. Larsson R, Runeson K. Discontinuous displacement approximation for capturing plastic localization. International Journal for Numerical Methods in Engineering 1993; 36:2087-2105. 
13. Jirasek M. Finite elements with embedded cracks. Internal Report 98/01, LSC-DGC, Ecole Polytecnique de Lausanne, April 1998.

14. Bazant Z et al. Continuum theory for strain softening. Journal of Engineering Mechanics ASCE 1984; 110:1666-1692.

15. Muhlhaus H. Application of Cosserat theory in numerical solutions of limit load problems. Ingenieur Archiv 1989; 59:124-137.

16. de Borst R, Muhlhaus H. Gradient dependent plasticity: formulation and algorithmic aspects. International Journal for Numerical Methods in Engineering 1992; 35:521-539.

17. Muhlhaus H, Aifantis E. A variational principle for gradient plasticity. International Journal of Solids and Structures 1991; 28:845-857.

18. de Borst R, Muhlhaus H. Computational strategies for gradient continuum models with a view to localisation of deformation. Proceedings of 4th International Conference on Non-linear Engineering Computations, Bicanic et al. (eds). Pineridge Press: Swansea, 1992; 239-260.

19. Chambon $\mathrm{R}$ et al. Etude de la localisation unidimensionelle à l'aide d'un modèle de second gradient. Comptes Readns del' Academic des Sciences 1996; 323:231-238.

20. Chambon $\mathrm{R}$ et al. One dimensional localisation studied with a second grade model. European Journal of Mechanics A/Solids 1998; 17:637-656.

21. Fleck NA, Hutchinson JW. Strain gradient plasticity. Advances in Applied Mechanics 1997; 33:295-361.

22. Fleck NA, Hutchinson JW. A discussion of strain gradient plasticity theories and application to shear bands. In Material Instabilities in Solids, de Borst R, van der Giessen E (eds). Wiley: Chichester 1998; 507-520.

23. Simo JC, Taylor RL. Tangent operators for elastoplasticity. Computer Methods in Applied Mechanics and Engineering 1985; 48:101-118.

24. Charlier R. Approche unifiee de quelques problemes non lineaires de mecanique des milieux continus par la methode des elements finis. Ph.D. Thesis, Universite de Liege, 1987.

25. Chambon R, Caillerie D, Desrues J, Crochepeyre S. A comparison of incremental behaviour of elastoplastic and CloE models. International Journal for Numerical and Analytical Methods in Geomechanics 1999; 23(4): $295-316$.

26. Desrues J, Chambon R. Shear band analysis for granular materials: the question of incremental non-linearity. Ingenieur Archiv 1989; 59:187-196.

27. Rice J. The localization of plastic deformation. In International Congress of Theoretical and Applied Mechanics, Koiter WD (ed.). North-Holland Publishing Company: Amsterdam, 1976.

28. Chambon R, Crochepeyre S, Desrues J. Localization criteria for non-linear constitutive equations of geomaterials. Mechanics of Cohesive-Frictional Materials 2000; 5:61-82.

29. Chambon R, Crochepeyre S, Charlier R. Numerical study of bifurcation of a biaxial test. Fifth International Conference on Computational Plasticity, Owen, Onate, Bhinton (eds). CIMNE, Barcelona 1997; 674-681.

30. Crochepeyre S. Contribution à la modélisation numérique et théorique de la localisation et de la post localisation dans les géomatériaux. Ph.D. Thesis, Universite Joseph Fourier Grenoble, 1998.

31. Stuart AM, Humphries AR. Dynamical Systems and Numerical Analysis. Cambridge University Press: Cambridge, 1996.

32. de Borst R. Bifurcations in finite element models with a non associated flow law. International Journal for Numerical and Analytical Methods in Geomechanics 1988; 12:99-116. 